\title{
Token Reinforcement of Verbal Responses Controlled by Temporally Removed Verbal Stimuli
}

\author{
Lee L. Mason ${ }^{1} \cdot$ Donald Davis ${ }^{1}$ - Alonzo Andrews ${ }^{1}$ \\ Published online: 18 March 2015 \\ (C) Association for Behavior Analysis International 2015
}

Establishing control by verbal stimuli over the listener with an autism spectrum disorder (ASD), of which circumscribed stimulus control is a defining feature (American Psychiatric Association 2013), may represent a formidable challenge to the typical verbal community. Without directed intervention, individuals with autism often respond indiscriminately to other people's verbal behavior (Pétursdóttir et al. 2008; Sundberg and Sundberg 2011). Verbal stimuli are particularly complex, because their effects on the environment are merely temporary. Once the speaker stops speaking, the verbal stimulus no longer exists. The complexity is compounded when the listener with autism is asked to recount events that happened in the past. Within such verbal episodes, the listener with autism may emit prepotent verbal responses under disproportionate environmental control (Kenny et al. 2014). Bíró and Russell (2001) define prepotent responses as "an erroneous response that is called out either by some salient feature of the environment or by some feature rendered salient through previous learning" (p. 98). For instance, when asked in the evening, "What did you eat for lunch today?", the listener with autism may reply with what he/she would like to eat for lunch tomorrow (disproportionate mand control), by listing food items presently on the dinner table (disproportionate tact control), or with partial echolalia (e.g., "Lunch today."; disproportionate echoic control). Consequently, the speaker's verbal stimulus fails to exact control over the listener with autism.

Token economies have been shown to be effective as a supplemental reinforcement strategy when the natural contingencies are too thin or inadequately conditioned to maintain a behavioral repertoire (Hackenberg 2009). Likewise, token reinforcement may augment the natural contingencies provided by the verbal community when conditioning the listener to respond effectively. The purpose of the current research was to establish control by temporally removed verbal stimuli over the intraverbal responding of children with ASD through the use of token reinforcement.

Lee L. Mason

lee.mason@utsa.edu

Donald Davis

dondavis@reglue.org

Alonzo Andrews

alonzo.andrews@utsa.edu

1 Department of Interdisciplinary Learning and Teaching, The University of Texas at San Antonio, San Antonio, TX, USA 


\section{Method}

\section{Participants}

Three male children diagnosed with ASD, one preadolescent and two adolescents, took part in this research. Each child displayed minor communication deficits and was therefore asked to participate in the current research project. Michael was 8 years old and diagnosed with Asperger's disorder and attention-deficit/hyperactivity disorder. Robert was 15 years old and diagnosed with both ASD and bipolar disorder. Thomas was 17 years old and diagnosed with ASD. Thomas also had a hearing impairment corrected through the use of a hearing aid in his right ear. All three children possessed an advanced verbal repertoire, placing above level 3 in all domains of the Verbal Behavior Milestones Assessment and Placement Program (Sundberg 2008). The participants demonstrated a balanced stimulus control ratio across four primary verbal operants (Mason and Andrews 2014), and could effectively respond to a battery of revolving wh- questions. Despite such verbal acuity, none of the participants engaged in sustained conversation (i.e., successive intraverbal exchanges), as they did nothing to catalyze social interactions (e.g., by manding for information from others).

\section{Setting}

The experiment took place in a university-based applied behavior analysis (ABA) center for children with autism. Throughout the study, participants worked one-onone with master's level ABA students who served as behavior technicians. For each participant, an individualized treatment plan primarily focused on reinforcing communication and social skills within the context of academic and vocational training.

Behavior analytic intervention was provided 4 days a week in 90-min increments. The behavior technicians were responsible for implementing the independent variable and collecting data on their assigned children. Although the participants often worked in small groups to address some of their treatment goals, all experimental procedures were carried out one-on-one, at a small table where the participant and behavior technician worked on other individual learning objectives.

\section{Response Definition and Measurement}

The frequency of each participant's discriminated intraverbal responses was used as the dependent measure across both phases of the study. Discriminated responses were defined as intraverbal behavior in response to the behavior technician's probe for information about the visitor that corresponded with the information provided by the visitor. For each session, the technicians used event recording to tally the number of visitor attributes discriminated by each participant.

Interobserver agreement (IOA) data were collected between the behavior technician who served as the primary data collector for her participant, and one of the researchers serving as a secondary data collector. Using a copy of each visitor's information sheet, data collectors independently checked off each item accurately discriminated by the participant. Trial reliability was calculated by dividing the number of agreements by the sum of agreements and disagreements, and then multiplying by 100 . Interobserver 
agreement was assessed for $44 \%$ of all sessions across participants and experimental conditions, and averaged $98 \%$ (range 90-100\%).

\section{Procedures}

Throughout the study, participants were introduced to strangers who visited the autism center, and with whom they had no prior contact. These visitors were university students and staff, both male and female, who ranged in age from 21 to 55 years. Only one visitor was systematically introduced to each participant per day, and all introductions took place privately at the participant's workstation. Up to three experimental sessions were run per day, one for each participant.

Prior to introducing themselves to the participants, visitors were asked to fill out information sheets about themselves. The information sheet asked for 20 specific attributes about the visitor, such as name, age, hometown, and preferred activities among others. Convention suggests that such mundane personal information is an appropriate subject of conversation when meeting someone unfamiliar, and is frequently taught in social skills curricula (e.g., Gresham and Elliott 2008).

The personal information provided by the visitor was then transferred onto laminated index cards that were scripted to convey two or three attributes about the visitor at a time to the participant. For instance, the first card read "Hi. My name is What's your name? It's nice to meet you. How old are you? I'm ___. . The visitor was given a total of ten scripted index cards to facilitate the conversation with each participant. For each conversation, the first card with the introduction and the last card with the closing statement remained constant. In between, the eight intermediate cards were shuffled to create a novel conversation for each visitor. Visitors were also told that they did not necessarily need to read or memorize each card exactly, so long as the pertinent information (i.e., personal attributes) on each card was provided in a concise and natural manner. The visitor was also given a digital audio recorder to archive the conversation with each participant for further analysis.

Upon completing the initial steps in the protocol described above, the visitor was escorted by one of the researchers to the table where the participant was working with the behavior technician. The researcher asked the behavior technician to excuse herself for a moment to complete some paperwork while the visitor met with the participant. The behavior technician sat down at a nearby table to listen in on the conversation while completing a fidelity of implementation checklist. As soon as the behavior technician stepped away, the visitor sat down in her place and began the conversation with the first index card.

Visitors were asked to convey the information on each index card to the participant, before moving to the next card. If the participant directed the conversation away from the script, the visitor was asked to respond accordingly and then redirect the conversation back to the written prompts. Furthermore, visitors were asked not to repeat any information on the cards, unless it was information for which the participant specifically asked. This format was repeated until all ten cards were complete, at which point the visitor terminated the conversation and left the observation room.

Immediately after the visitor departed, the behavior technician returned to the table and asked the participant for information about the visitor. Probes began within $1 \mathrm{~min}$ of the visitor's departure, however, depending on the length of the conversation (range 6:24 to $8: 08 \mathrm{~min}$ ) and the order of the cards, some visitor attributes were more temporally 
removed than others. Each probe began by asking the participant, "Who was that person to whom you were just speaking?" or a slight derivation thereof. Holding the information sheet filled out by the visitor so that the participant could not read it, the behavior technician then tallied the number of discriminated responses provided by the participant.

A free-operant stimulus preference assessment was conducted at the start of each day's session to identify potential rewards with which to reinforce discriminated responses (Roane et al. 1998). An individualized token economy system was employed with each participant, through which he could earn points that were later backed up with access to his reward of choice. Michael consistently chose access to LEGO $^{\circledR}$ toys as his preferred reward. Robert alternated between visits to the campus police station or library, and access to a computer. Thomas always exchanged points directly for shots on the basketball goal. The token economy was established and conditioned for 3 weeks prior to collecting baseline data. The number of tokens corresponded to the duration of the reward activity. Participants were able to exchange their tokens at the end of each day's session, however, the duration of the activity varied according to the number of points earned.

In the current study, treatment consisted of immediately delivering a token to the participant for each discriminated response. The behavior technicians were limited to three general probes for information (e.g., "Can you tell me anything else about him?"), but were directed not to ask for specific information (e.g., "Did he have any pets?"). The session was terminated after the third probe. Any information later recalled by the participant was not included for data collection purposes. The behavior technicians were not blind to the experimental conditions, as they served as both primary data collector and contingency manager.

\section{Experimental Design}

The experiment employed a concurrent multiple-baseline design across participants. The onset of treatment was staggered for each participant to establish multiple points of comparison across two phases:

No tokens. Baseline data were collected using the protocol described above. In this phase, responses from the participants were tallied, but not rewarded. Five baseline data points were collected for Michael, nine for Robert, and 14 for Thomas.

Tokens. After stable responding was observed for each participant, contingent token rewards were applied. Using a continuous reinforcement schedule, participants earned a token for each visitor attribute accurately reported.

\section{Fidelity}

Procedural fidelity was monitored across both visitors and behavior technicians. The procedures described above were broken down into a 20 -item checklist for the purpose of collecting fidelity data. Procedural fidelity was assessed every day of the study for $47 \%$ of all sessions. Fidelity of implementation averaged $95 \%$ across all observations, ranging from 89 to $100 \%$. After each session in which fidelity fell below $100 \%$, behavior technicians and/or visitors were informed of the procedural violation and provided with specific instruction on how to address it for the subsequent session. 


\section{Results and Discussion}

The effects of token reinforcement on the frequency of participants' discriminated intraverbal responses under the control of temporally removed verbal stimuli can be seen in Fig. 1. Baseline responding was relatively low for Robert and Thomas; the majority of sessions yielded no responses, with no more than one discriminative response from Robert and two from Thomas. Michael's responding was more consistent, with three to four responses throughout much of the baseline phase.

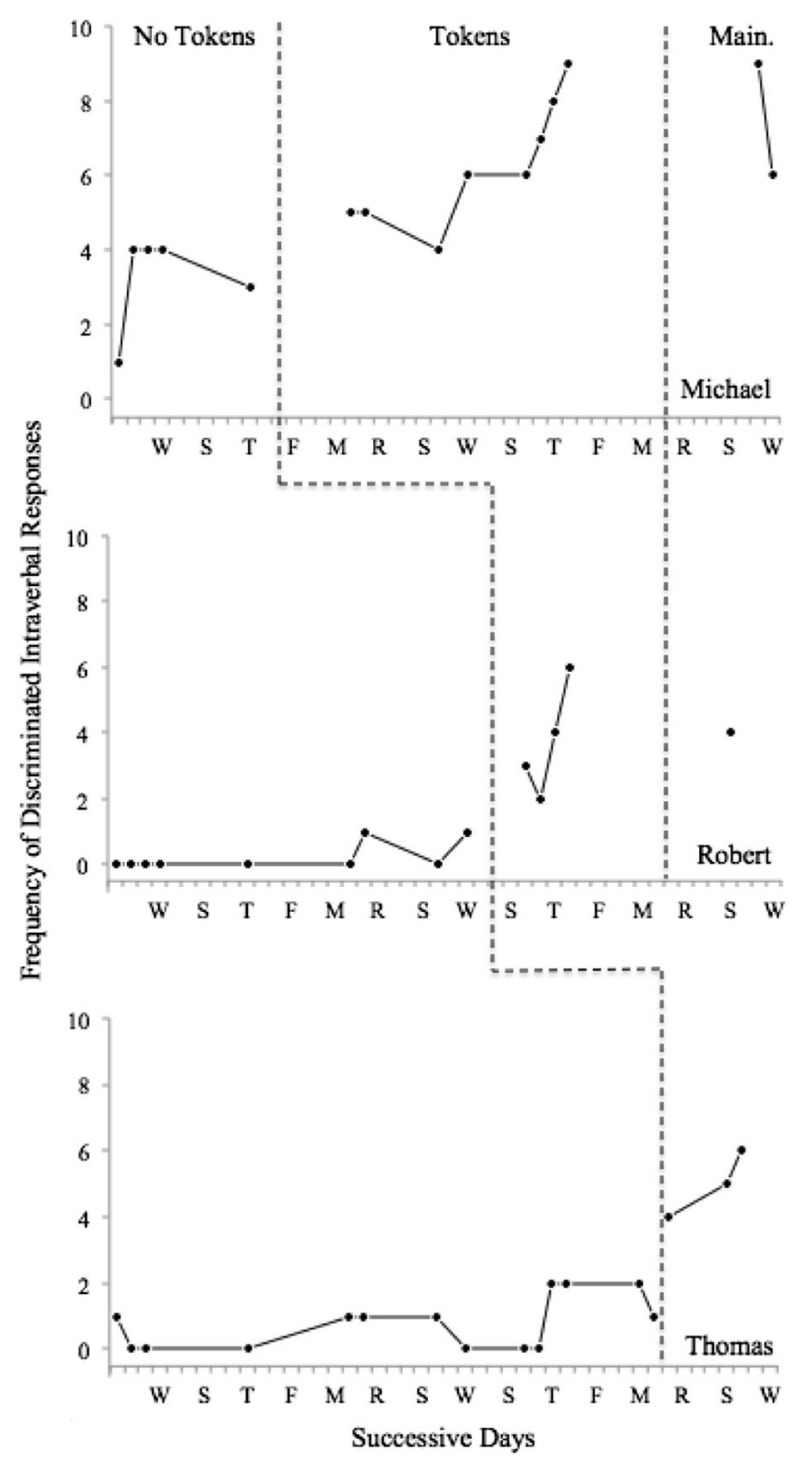

Fig. 1 The effects of token reinforcement on participants' discriminated intraverbal responses under the multiple control of immediate probes for information by behavior technicians and the temporally removed verbal stimuli of visitors 
All three participants showed an increase in level and trend only after the tokens were applied. Michael averaged 3.0 discriminated responses during baseline and 6.25 discriminated responses after employing the token economy system. Robert averaged 0.25 discriminated responses before tokens were applied and 3.75 discriminated responses during treatment. Thomas averaged 0.85 discriminated responses during baseline and 4.66 discriminated responses during treatment. Additionally, an acceleration in the frequency of discriminated responses is apparent throughout the treatment phase for each participant.

Maintenance of discriminated responses was assessed for both Michael and Robert. After sufficient treatment data had been gathered to demonstrate an effect, Michael and Robert were placed on a 2-week maintenance hiatus. During this time, they were not introduced to any visitors to the center. After 2 weeks, identical treatment proceduresincluding token reinforcement-were once again employed with novel visitors. Follow-up probes for both Michael and Robert showed that they maintained treatment levels of discriminated intraverbal responses. An extended baseline for Thomas precluded the addition of maintenance assessment for Thomas prior to the conclusion of the study.

Visual analyses of the data demonstrate a functional relationship between the use of token reinforcement and the frequency of discriminated responses of participants with autism. A discernable change in both level and trend was evident only after the intervention was implemented with each participant. A slight practice effect can be discerned throughout the baseline phase for each participant in Fig. 1, but along with the introduction of the token economy system comes a clear increase in discriminated responding. Additionally, the results were maintained over time; an important effect to differentiate the strength of the token economy system alone. The effects of repeated practice were mitigated by the 2 weeks prior to maintenance.

A review of the transcribed recordings of the conversations with visitors revealed that participants were more likely to emit responses under faulty stimulus control after the implementation of token reinforcement. Only ten total prepotent responses $(7 \%$ of all participant responses) were recorded throughout the study, seven of which occurred during the intervention phase. In contrast, participants were less likely to emit any response during baseline. Robert emitted one prepotent response after the tokens were employed, while Thomas emitted the majority of prepotent responses (nine total, three during baseline and six throughout the intervention). Namely, across both phases of the study, the source of strength that frequently controlled Thomas's response to the visitor's favorite type of music came from his own favorite type of music.

Michael demonstrated the greatest number of correct discriminated intraverbal responses during intervention; nine correct responses out of a possible 20. A social comparison would have been informative to this investigation as a measure of social validity. Future researchers may seek to address this limitation by conducting a similar protocol with typically developing same-age peers to gather normative data on this variable that could then be compared against participants with autism.

In conclusion, the token economy system employed here was effective to bring the participants' verbal behavior under the convergent control of both the technician's probe and the temporally removed verbal behavior of the visitor. Importantly, discriminated responding was not readily observed until the required contingency was 
established. Explicit conditioning was required to bring the verbal responding of each participant under the control of the visitor's temporally removed verbal stimuli. Future research should extend the current study to explore pairing token reinforcement with more natural contingencies, and thinning the schedule of reinforcement to match that provided by the verbal community.

Acknowledgments Special thanks to Stephanie Curtis, Hilary McCown, and Rachael Trevino for their assistance in completing this research.

\section{Appendix: Sample Script}

"Hey, my name's . What's your name? It's nice to meet you. How old are you? I'm

$<10$-second pause. $>$

"Where are you from? I'm from _. I grew up (t)here with my brother(s) and sister(s). Do you have any brothers and sisters?"

$<10$-second pause. $>$

"Do you have any pets? I have a pet Its name is

$<10$-second pause. $>$

"Do you like to watch ? My favorite team is the Do you have a favorite team?"

$<10$-second pause. $>$

"Do you like food? My favorite restaurant is called Have you been there? They have the best ."

$<10$-second pause. $>$

"Have you seen any good movies recently? I love movies. My favorite is

$<10$-second pause. $>$

"What kind of music do you like to listen to? I like favorite song of theirs is ," Have you heard of ? My

$<10$-second pause. $>$

"Do you like to read? My favorite book is It was written by

$<10$-second pause. $>$

"What do you want to be when you grow up? Some day I'd like to be a , but when I'm not at work, I'd like to go to

$<10$-second pause. $>$

"Well, it was nice to meet you. Take care!" 


\section{References}

American Psychiatric Association. (2013). Diagnostic and statistical manual of mental disorders (5th ed.). Washington, DC: Author.

Bíró, S., \& Russell, J. (2001). The execution of arbitrary procedures by children with autism. Development and Psychopathology, 13, 97-110.

Gresham, F. M., \& Elliott, S. N. (2008). Social skills improvement system: rating scales. Bloomington: Pearson Assessments.

Hackenberg, T. D. (2009). Token reinforcement: a review and analysis. Journal of the Experimental Analysis of Behavior, 91, 257-286.

Kenny, N., Devlin, S., Barnes-Holmes, D., Barnes-Holmes, Y., \& Stewart, I. (2014). Competing arbitrary and nonarbitrary stimulus relations: the effects of exemplar training in adult participants. The Psychological Record, 64, 53-61.

Mason, L. L., \& Andrews, A. (2014). Referent-based verbal behavior instruction for children with autism. Behavior Analysis in Practice, 7, 107-111.

Pétursdóttir, A. I., Carr, J. E., Lechago, S. A., \& Almason, S. M. (2008). An evaluation of intraverbal training and listener training for teaching categorization skills. Journal of Applied Behavior Analysis, 41, 53-68.

Roane, H. S., Vollmer, T. R., Ringdahl, J. E., \& Marcus, B. A. (1998). Evaluation of a brief stimulus preference assessment. Journal of Applied Behavior Analysis, 31, 605-620.

Sundberg, M. L. (2008). Verbal behavior milestones assessment and placement program: the VB-MAPP. Concord: AVB Press.

Sundberg, M. L., \& Sundberg, C. A. (2011). Intraverbal behavior and verbal conditional discriminations in typically developing children and children with autism. The Analysis of Verbal Behavior, 27, $23-43$. 